\title{
IDENTIFICACION DE CAOS EN SERIES TEMPORAIS MICROMETEOROLÓGICAS MEDIDAS EN EL MATO GROSSO - BRASIL
}

\section{Geison Jader Mello ${ }^{1}$, Marcelo Sacardi Biudes ${ }^{2}$, Raphael Souza Rosa Gomes ${ }^{3}$, Tonny Jader de Moraes ${ }^{4}$}

${ }^{1}$ Doctor en Física Ambiental, Instituto Federal de Educación, Ciencia y Tecnología de Mato Grosso - Campus Cuiabá Cel. - Octayde Jorge da Silva, Cuiabá-MT, Brasil.

E-mail: geison.mello@cba.ifmt.edu.br

${ }^{2}$ Doctor en Agricultura Tropical, Universidad Federal de Mato Grosso, Cuiabá-MT, Brasil.

${ }^{3}$ Doctor en Física Ambiental, Universidad Federal de Mato Grosso, Cuiabá-MT, Brasil.

${ }^{4}$ Doctor en Física Ambiental, Universidad Federal de Mato Grosso, Cuiabá-MT, Brasil.

Recebido em: 04/10/2019 - Aprovado em: 30/11/2019 - Publicado em: 15/12/2019 DOI: 10.18677/EnciBio_2019B32

\begin{abstract}
RESUMEN
Se sabe que las series temporales medidas en sistemas microclimáticos no son ni aleatorias y ni siguen un patrón lineal, de forma que es coherente afirmar que los datos poseen más estructuras que los métodos tradicionales y las herramientas lineales son capaces de capturar, especialmente porque la no linealidad es inherente a los ecosistemas. En este sentido, el objetivo de este trabajo fue identificar el comportamiento no lineal de series temporales de saldo de radiación, temperatura y humedad del aire medidas por encima del dosel en bosque estacionalmente inundado en el Pantanal Matogrossense a partir de la Teoría de los Sistemas dinámicos. Se aplicaron pruebas de no linealidad que justifican el enfoque, como la prueba Surrogate (SUR) que sirve para distinguir entre la no linealidad y la aleatoriedad de series temporales. Los resultados fueron que las tres series temporales micrometeorológicas de Saldo de Radiación, Temperatura del Aire y Humedad Relativa del Aire medidas en el Pantanal Norte de Mato Grosso, Brasil fueron probados en términos de no linealidad y obtuvieron resultados positivos. Esto caracteriza que la serie temporal es no lineal y no aleatoria, y se puede entonces, con seguridad echar mano del marco teórico de la teoría del caos para el modelado.
\end{abstract}

PALABRAS CLAVE: identificación de caos, prueba de no linealidad, dinámica no lineal, teoría del caos.

\section{IDENTIFICATION OF CHAOS IN TEMPORARY MICROMETEOROLOGICAL SERIES MEASURES IN MATO GROSSO - BRAZIL}

\begin{abstract}
It is known that the time series measurements in microclimatic systems are neither random nor follow a linear pattern, so that it is consistent state that the data have more structure than traditional methods and the linear tools are able to capture, especially as nonlinearity is inherent to ecosystems. In view of this, the objective of this study was to identify the non-linear behavior of time series of net radiation, temperature and humidity
\end{abstract}


of the air above the canopy measured in seasonally flooded forest in Mato Grosso Pantanal from the theory of dynamical systems. nonlinearity tests were applied justifying the approach such as Surrogate Test (SUR) which serves to distinguish between the nonlinearity and the randomness of time series. The results were that the three time series Micrometeorological Radiation Balance, Air Temperature and Relative Humidity measurements in North Pantanal Mato Grosso, Brazil, were tested for nonlinearity and obtained positive results. It features that the time series is not linear and not random, and can then safely resort to the theoretical framework of chaos theory for modeling.

KEYWORDS: chaos identification, nonlinearity test, nonlinear dynamics, chaos theory.

\section{INTRODUCCIÓN}

El Pantanal ocupa el lugar de mayor planicie tropical de inundación estacional del planeta, abriendo biodiversidad desproporcionadamente alta y distinguiendo por fauna y flora peculiar (FERREIRA-JUNIOR et al., 2016). Este status permite desempeñar un papel muy importante en la estabilidad dinámica del clima regional

Las investigaciones recientes encontraron indicios de alteración en el ciclo hidrológico del norte del Pantanal (PENATTI et al., 2014). Esto se caracterizó por el aumento de los picos de precipitación máxima, sugiriendo que ese ciclo está siendo afectado por los cambios climáticos. También hay un rápido cambio en la vegetación en campos estacionalmente inundados que también ha sido considerado un efecto de cambios climáticos ocurridos en ciclos plurianuales (VOURLITIS et al., 2015).

La deforestación es el mayor estigma de la actividad antrópica insertada en la dinámica de la ocupación del espacio del Pantanal (ALMEIDA et al., 2015;). Con el objetivo de ampliar las áreas destinadas a la agricultura y la ganadería, la deforestación ejerce impactos negativos junto al ecosistema local causando desequilibrio sistémico (PARANHOS FILHO et al., 2014), a ejemplo de lo que ocurre en la Reserva Particular del Patrimonio Natural (RPPN) SESC Pantanal Mato-grossense que viene sufriendo invasión de una vegetación mono-dominante llamada Cambará (Vochysia divergos Pohl).

Se sabe que con el cambio de la cobertura vegetal original por áreas de pasturas y cultivos, el flujo de vapor de agua a la atmósfera es alterado, así como la reflectividad del suelo, afectando directamente el saldo de radiación, entre otros efectos (BIUDES et al., 2016). Pero todavía no se entienden claramente cómo estas alteraciones regionales en el uso del suelo pueden afectar el ciclo hidrológico y el régimen de lluvias en la región. Uno de los motivos es la falta de series históricas de datos micrometeorológicos medido in situ para detectar y monitorear estos posibles cambios en el clima regional. De esta forma es de suma importancia comprender y hacer previsiones de la dinámica de variables micrometeorológicas como el saldo de radiación, la temperatura y la humedad relativa del aire, y sus relaciones ante el escenario de los cambios climáticos locales y globales a corto y largo plazo.

Se sabe que las series temporales medidas en estos sistemas microclimáticos no son ni aleatorias y no siguen un patrón lineal, de forma que es coherente afirmar que los datos poseen más estructuras que los métodos tradicionales y las herramientas lineales son capaces de capturar, especialmente porque la no linealidad es inherente a los ecosistemas (MELLO et al., 2013a, 2013b). De hecho, la descripción micrometeorológica de ecosistemas, como los bosques tropicales estacionalmente inundados, visto su no linealidad, el número y la variedad de subsistemas en interacción, así como los efectos antropogénicos, es un problema expresivo (PAULO et al., 2015). 
Los ecosistemas son sistemas abiertos, dinámicos y complejos y su estudio necesita herramientas que sean capaces de capturar la dinámica de las series temporales medidas en el bosque y también que puedan contribuir con proyecciones futuras (BUSTAMANTE et al., 2016). Los avances en esta dirección se han reportado con la aplicación de herramientas adecuadas para comprender y predecir la dinámica de las series temporales medidas en las flores tropicales.

Se destacan las investigaciones que analizan la posible naturaleza no lineal de los datos ambientales, contribuyendo para el entendimiento y modelado de la interacción biosfera-atmósfera. En la teoría de los sistemas dinámicos, varias investigaciones involucrando series temporales de diferentes variables ambientales mencionan la posible existencia de atractores climáticos de baja dimensión fractal e indican el número mínimo de variables presentes en la evolución de la dinámica del sistema (PAULO et al., 2015). Es decir, un sistema caótico que exhibe el comportamiento de un sistema dinámico que no ha evolucionado de acuerdo con una ley determinista y se rige por ecuaciones cuyas soluciones son extremadamente precisas en las condiciones iniciales, de modo que pequeñas diferencias conducen a estados posteriores extremadamente diferentes.

En esta perspectiva el objetivo general de este trabajo fue entender el comportamiento micrometeorológico del Pantanal a partir del análisis de las variables micrometeorológicas saldo de radiación, temperatura y humedad relativa del aire buscando predecir este comportamiento por medio de la teoría de sistemas dinámicos (teoría del caos)

Para ello, se definieron los siguientes objetivos específicos:

Analizar las series temporales micrometeorológicas, y,- identificar el comportamiento no lineal de series temporales a partir de la Teoría de los Sistemas dinámicos aplicando la prueba de no linealidad que justifica el abordaje, tal como la Prueba Surrogate (SUR) que sirve para distinguir entre la no linealidad y la aleatoriedad de series temporales

\section{MATERIAL E MÉTODOS}

Localización y caracterización del área de estudio. El sitio experimental se localiza en la Reserva Particular del Patrimonio Natural - RPPN SESC - Pantanal, municipio de Barón de Melgaço - MT, distante 160 km de Cuiabá, Mato Grosso, en la región Centro-Oeste de Brasil, donde está instalada una torre micrometeorológica de. $32 \mathrm{~m}$ de altura (1639'50 "S, 5647'50"O, $120 \mathrm{~m}$ ) (Figura 19). Esta zona presenta un bosque monodominante de Cambará (Vochysia divergentes), conocida localmente como cambarazal, con altura del dosel variando entre 28 a 30 m (ARIEIRA; NUNES DE CUNHA, 2006). El suelo se clasifica como GLEISSOLO HÁPLICO Ta Distrófico. EI clima es Aw según la clasificación de Köppen, cuya media anual de temperatura del aire es de $25,6^{\circ} \mathrm{C}$ y la humedad relativa del aire es del $73,1 \%$, la precipitación media anual es de $1342 \mathrm{~mm}$, con una precipitación inferior a 15 en el período seco (junio a agosto) y estación lluviosa con el $97 \%$ de la precipitación acumulada anual (BIUDES et al., 2009, INMET, 2009). A menudo la inundación ocurre entre diciembre y marzo (0,6 $1,5 \mathrm{~m}$ de altura) caracterizando la fase acuática del Pantanal, que acompaña la estación lluviosa y la oscilación anual del nivel de agua del río Cuiabá, siendo altamente influenciada por la precipitación (NUNES DE CUNHA ; JUNK, 2004; ARIEIRA ; NUNES DE CUNHA, 2006). 


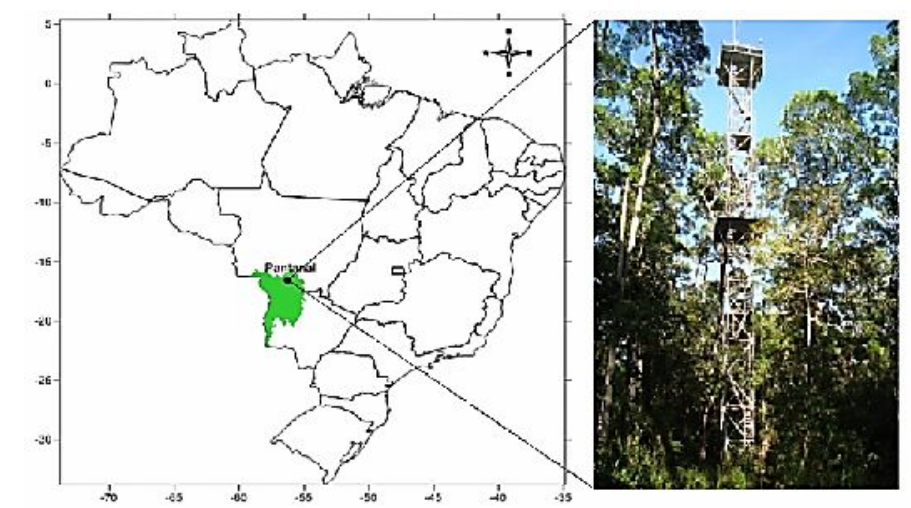

FIGURA 1: Mapa de Brasil, con destaque para el Pantanal Brasileño, localización del área de vegetación monodominante de Cambará en la RPPN SESC y de la torre micrometeorológica.

Período de estudio. Los datos utilizados en este trabajo fueron muestreados cada 10 segundos con extracción de promedios cada 15 minutos, en el período comprendido del 1 de septiembre de 2006 hasta el 1 de septiembre de 2010.

Recolección de datos. La instrumentación utilizada para la recolección de datos de saldo de radiación (Rn) fue un Net Radiometer, Kipp \& Zonen Delft, Inc., Holland; para la temperatura $(\mathrm{T})$ y humedad relativa $(\mathrm{Rh})$ un termohigrómetro (HMP $45 \mathrm{C}$, Vaisala, Inc., Helsinki, Finlandia) instalados a 37,7 m. Los datos fueron procesados y almacenados por dataloggers (CR 10X, Campbell Scientific, Inc., Ogden, Utah, USA). Para aumentar el número de canales de entrada en el registrador se utilizó una tarjeta multiplexora (AM16 / 32A-ST-SW, Campbell Scientific, Inc., Ogden, Utah, USA).

Análisis de Series Temporais. Se analizaron tres series temporales experimentales micrometeorológicas: del saldo de radiación $(\mathrm{Rn})$, de la temperatura del aire $(\mathrm{T})$ y de la humedad relativa $(\mathrm{Rh})$ recogida en el sitio experimental. El programa surrogates.exe del paquete de software TISEAN - Nonlinear Time Series Analysis (software de dominio público disponible en http://www.mpipksdresden.mpg.de/ tisean/) se utilizó para probar la no linealidad de las series temporales utilizado.

\section{RESULTADOS Y DISCUSIÓN}

Los resultados inician por la descripción microclimática de las series temporales medidas en el área de estudio. A continuación se aplican pruebas de no linealidad en las series temporales medidas y reconstruido el espacio de fase -dimensional en el cual se suman el conjunto atractor de la dinámica de cada serie temporal. Los proveedores de un atractivo, se realizan análisis cualitativos y cuantitativos de su dinámica para estimar los parámetros necesarios al modelo en cuanto a sus especificidades. Por último, y como propósito principal de este trabajo, se presentan las series temporales modeladas por predicción no lineal simple y los errores de predicción en términos de comparación con las series temporales medidas.

Descripción Microclimática. En esta sección se hace la descripción micrometeorológica del área de estudio en términos de las variables medidas y de la estacionalidad (Tabla 1). El saldo de radiación fue significativamente afectado por las estaciones del año. El saldo de radiación tuvo mayor media \pm desviación estándar 
durante la estación lluviosa $(11,37 \pm 4,06)$ que durante la estación seca $(7,40 \pm 1,97)$. Esta diferencia es esperada debido al factor astronómico, pues la estación lluviosa ocurre en el período de mayor incidencia de radiación solar en el hemisferio sur.

TABLA 1: Media mensual y estacional y desviación estándar mensual y estacional del saldo de radiación $(R n)$, de la temperatura del aire $(T)$ y de la humedad relativa $(R h)$.

\begin{tabular}{|c|c|c|c|}
\hline Variável/mês & $R n\left(M / m^{-2} \operatorname{dia}^{-1}\right)$ & $T\left({ }^{\circ} \mathrm{C}\right)$ & $R h(\%)$ \\
\hline Enero & $13,10 \pm 3,95$ & $26,57 \pm 1,39$ & $80,79 \pm 8,10$ \\
\hline Febrero & $12,98 \pm 4,22$ & $26,82 \pm 1,32$ & $82,21 \pm 5,51$ \\
\hline Marzo & $12,65 \pm 3,71$ & $27,32 \pm 1,40$ & $81,30 \pm 5,54$ \\
\hline Abril & $10,44 \pm 2,88$ & $26,62 \pm 1,97$ & $79,48 \pm 6,58$ \\
\hline Mayo & $7,85 \pm 2,32$ & $23,64 \pm 3,61$ & $76,45 \pm 7,81$ \\
\hline Junio & $6,65 \pm 1,41$ & $22,92 \pm 3,09$ & $71,69 \pm 9,96$ \\
\hline Julio & $6,85 \pm 1,87$ & $23,42 \pm 4,04$ & $61,44 \pm 12,49$ \\
\hline Agosto & $8,65 \pm 1,88$ & $25,02 \pm 3,66$ & $54,02 \pm 14,24$ \\
\hline Septiembre & $9,19 \pm 3,05$ & $26,43 \pm 3,63$ & $57,33 \pm 13,85$ \\
\hline Octubre & $10,88 \pm 4,11$ & $27,08 \pm 2,14$ & $71,67 \pm 10,10$ \\
\hline Noviembre & $12,70 \pm 3,84$ & $27,09 \pm 1,81$ & $75,21 \pm 9,22$ \\
\hline Deciembre & $12,57 \pm 4,15$ & $26,42 \pm 1,35$ & $80,69 \pm 8,39$ \\
\hline Anual & $10,37 \pm 4,04$ & $25,78 \pm 3,07$ & $72,63 \pm 13,73$ \\
\hline Seca & $7,40 \pm 1,97$ & $23,79 \pm 3,73$ & $62,27 \pm 14,35$ \\
\hline Lluvia & $11,37 \pm 4,06$ & $26,79 \pm 2,49$ & $76,13 \pm 11,38$ \\
\hline
\end{tabular}

La temperatura del aire fue significativamente afectada por las estaciones del año. Las medias mensuales de la temperatura \pm desviación estándar fueron mayores durante la estación lluviosa $(26,79 \pm 2,49)$ que estación seca $(23,79 \pm 3,73)$. Los mayores valores de las temperaturas medias durante la estación lluviosa están relacionados con la mayor disponibilidad de energía radiante. La reducción en los valores de la temperatura del aire en la estación seca, además de la menor disponibilidad de energía radiante, puede haber sido resultado de la ocurrencia de frentes fríos. En estas situaciones de frentes fríos, ocurren caídas bruscas de la radiación incidente, debido a la presencia de nebulosidad. Durante estos eventos, la temperatura máxima puede ser 10 más baja que la temperatura máxima del día anterior. Además, dependiendo del volumen de aire frío, la temperatura puede tardar de 2 a 3 días para regresar a los valores registrados antes del paso del frente frío (FISCH, 1996, BIUDES et al., 2012).

La humedad relativa del aire fue significativamente afectada por las estaciones del año. Los promedios mensuales de la humedad relativa \pm desviación estándar fueron mayores durante la estación lluviosa $(76,13 \pm 11,38 \%)$ que en la estación seca $(62,27 \pm 14,35 \%)$. El aumento de la humedad relativa del aire en la estación lluviosa fue resultado de la mayor presencia de vapor de agua en el aire, asociado a la mayor precipitación (ALVES et al., 1999, BIUDES et al., 2012). Los menores valores de humedad relativa del aire en la estación seca están relacionados con la menor disponibilidad de agua en el suelo, lo que causa disminución en la evapotranspiración y consecuentemente en el flujo de calor latente (WRIGHT et al., 1996; BIUDES et al., 2009; BIUDES et al., 2012). 
La Figura 2 ilustra, a partir de medias mensuales de septiembre de 2006 hasta agosto de 2010, el patrón similar de variabilidad estacional de las variables. Se destacan los menores promedios de junio y julio, y las mayores de noviembre a marzo. A también exhibió los menores valores en junio y julio, y los mayores valores de septiembre hasta abril. Por fin la exhibieron los menores valores en el período más lejano de la estación lluviosa, es decir, julio, agosto y septiembre.

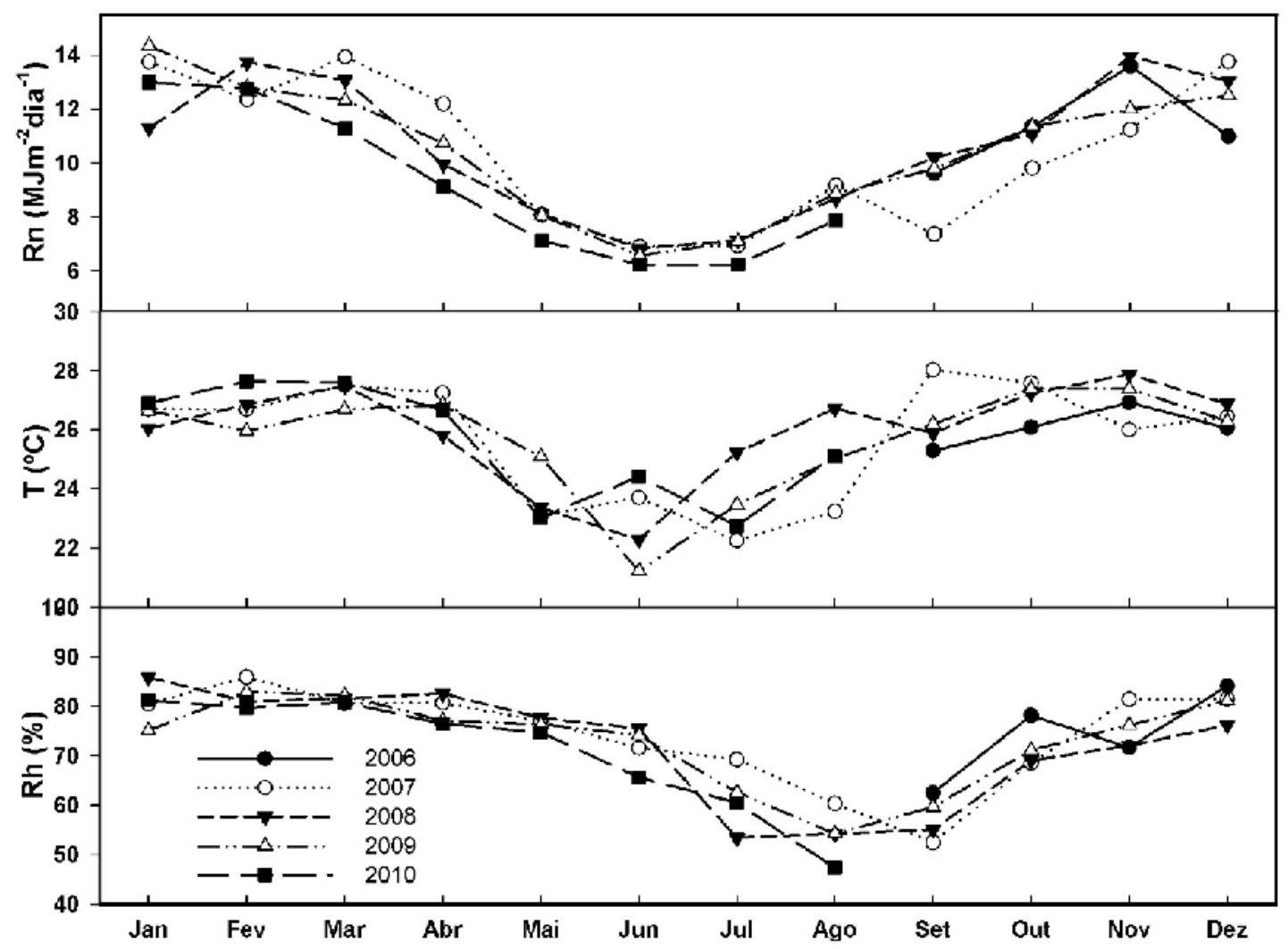

FIGURA 2: Media mensual del saldo de radiación (Rn), de la temperatura del aire $(\mathrm{T})$ y de la humedad relativa $(\mathrm{Rh})$.

Los valores mayores de los promedios de la temperatura del aire y humedad relativa en la estación lluviosa también son debidos al efecto liberador / moderador del término de almacenamiento de energía de la biomasa, o sea, durante la noche, la liberación de energía por la biomasa del bosque actúa en el sentido de mantener el balance radioactivo nocturno y no permite enfriamiento acentuado de la superficie (FISCH, 1996; BIUDES et al., 2012).

La precipitación en este período, según Biudes et al. (2013), fue significativamente afectada por las estaciones del año, con menores valores durante la estación seca reflejando el $6 \%$ y mayores valores en la estación lluviosa con el $94 \%$ del total precipitado.

Test de No Linearidad. Como justificación para el enfoque por teoría de los sistemas dinámicos se aplicó la prueba Surrogate en los datos antes del análisis y de la predicción (SCHREIBER \& SCHMITZ, 1996; SCHREIBER, 1999; MILLÁN et al., 2009). Los resultados de la aplicación de la prueba Surrogate (Tabla 2) se presentan en términos de número de interacciones y relativa discrepancia entre la serie temporal original y el conjunto de datos sustitutivos (Surrogate). Se admitió la hipótesis nula como la serie temporal fue generada por un proceso estacionario estocástico 
gaussiano, de forma que la estructura no lineal y sus invariantes topológicos aún se conservan en el Surrogate (SCHREIBER \& SCHMITZ, 2000).

TABLA 2- Parámetros para la construcción de las series Surrogate

\begin{tabular}{ccc}
\hline Variables Microclimáticas & № de Interacción & Discrepancias Relativa \\
\hline $\mathrm{Rn}$ & 766 & 0,2 \\
$\mathrm{~T}$ & 32 & 0,0029 \\
$\mathrm{Rh}$ & 204 & 0,0093 \\
\hline
\end{tabular}

Las series temporales de las variables $R n$ e a $R_{n(s u r)}$ la presentaron los mayores valores de número de interacciones y de discrepancia relativa. Muy posiblemente esto se debe al hecho de que el saldo de radiación es extremadamente sensible a cualquier variación de otras variables microclimatológicas, tales como nebulosidad o lluvia, cobertura del suelo y humedad del suelo, cubierta del dosel; a la estacionalidad; de manera que incluso derivando de un forzante lineal como radiación solar, esos aspectos hacen el Rn una dinámica diurna extremadamente no lineal en determinados períodos del año. Sin embargo, durante el período nocturno, o $\mathrm{Rn}$ el exhibe una dinámica bien comportada con patrones que flotan cerca de $-50 \mathrm{~W} \cdot \mathrm{m}^{2}$ durante casi toda la noche y durante casi todo el año. Esto justifica fuertemente, que aunque la serie tiene un exponente de Lyapunov positivo, indicando caos durante el día, también posea fragmentos con dinámica comportada durante la noche.

Recordando que un sistema caótico que exhibe el comportamiento de un sistema dinámico que no ha evolucionado de acuerdo con una ley determinista y se rige por ecuaciones cuyas soluciones son extremadamente precisas en las condiciones iniciales, de modo que pequeñas diferencias conducen a estados posteriores extremadamente diferentes.

Dada la complejidad del sistema ambiental Pantanal, con su alto número de variables que interactúan, los resultados teóricos de este trabajo apuntan a un enfoque con una teoría robusta apropiada para esta aplicación: la teoría de los sistemas dinámicos. Esta teoría está a la vanguardia de la investigación en ciencias ambientales como la física ambiental, la biometeorología y las ciencias atmosféricas.

\section{CONCLUSIONES}

Las tres series temporales micrometeorológicas de Saldo de Radiación, Temperatura del Aire y Humedad Relativa del Aire medidas en el Pantanal Norte de Mato Grosso, Brasil fueron analizadas y predichas por un abordaje fundamentado en la Teoría de los Sistemas Dinámicos. Las series temporales no se sometieron a ningún tipo de filtrado. Los cuatro años de datos micrometeorológicos del Saldo de Radiación, de la Temperatura del Aire de la Humedad Relativa del Aire, se probaron en términos de no linealidad y obtuvieron resultados positivos

De esta forma, se concluye que el abordaje por teoría de los sistemas dinámicos para análisis de no lineales se presenta útil cuando se aplican en datos micrometeorológicos

\section{AGRADECIMIENTOS}

A Pró-reitora de Pesquisa do IFMT através do Edital 034/2016 - DPI/PROPES/IFMT. 


\section{REFERENCIAS}

ALVES, F. S. M.; FISCH, G.; VENDRAME, I. F. Modificações do microclima e regime hidrológico devido ao desmatamento na Amazônia: um estudo de caso em Rondônia (RO), Brasil. Acta Amazônica, v.29, n.3, p.395-409, 1999. http://www.scielo.br/scielo.php?pid=S0044-59671999000300395\&script=sci_abstract

ARIEIRA, J.; NUNES DA CUNHA, C. Fitossociologia de uma floresta inundável monodominante de Vochysia divergens Pohl (Vochysiaceae), no Pantanal Norte, MT, Brasil. Acta Botanica Brasilica, v.20, p.569-580, 2006. http://www.scielo.br/scielo.php?script=sci_abstract\&pid=S0102-

$33062006000300007 \&$ Ing=en\&nrm=iso\&tlng=pt

BIUDES, M. S.; CAMPELO JUNIOR, J. H.; NOGUEIRA, J. S.; SANCHES, L. Estimativa do balanço de energia em cambarazal e pastagem no norte do Pantanal pelo método da razão de Bowen. Revista Brasileira de Meteorologia, v.24, n.2, p.135-143, 2009. http://www.scielo.br/scielo.php?script=sci_arttext\&pid=S010277862009000200003

BIUDES, M. S.; MACHADO, N. G.; DANELICHEN, V. H. M.; SOUZA, M. C.; VOURLITIS, G. L.; et al. Ground and remote sensing-based measurements of leaf area index in a transitional forest and seasonal flooded forest in Brazil. International Journal of Biometeorology, v.58, n.224, p.1-13, 2013. https://www.ncbi.nlm.nih.gov/pubmed/23943204

BIUDES, M. S.; NOGUEIRA, J. S.; DALMAGRO, H. J.; MACHADO, N. G.; DANELICHEN, V. H. M.; et al. Mudança no microclima provocada pela conversão de uma floresta de cambará em pastagem no norte do Pantanal. Revista de Ciências $\begin{array}{llll}\text { Agro-Ambientais, } & \text { v.10, } & \text { p.61-68, }\end{array}$ http://www.unemat.br/revistas/rcaa/docs/vol10/ARTIGO_7_RCAA_v10n1a2012.pdf

BUSTAMANTE, M. M., ROITMAN, I., AIDE, T. M., ALENCAR, A., ANDERSON, L. O., et al. Toward an integrated monitoring framework to assess the effects of tropical forest degradation and recovery on carbon stocks and biodiversity. Global Change Biology, v.22, no.1, 92-109, 2016. https://www.ncbi.nlm.nih.gov/pubmed/26390852

FERREIRA-JÚNIOR, W. G.; SCHAEFER, C. E.G.R.; CUNHA, C. N.; DUARTE, T. G.; CHIEREGATTO, L. C.; et al. Flood regime and water table determines tree distribution in a forest-savanna gradient in the Brazilian Pantanal. Anais da Academia Brasileira de Ciências, $\quad$ v. $88, \quad$ p. 2016. http://www.scielo.br/scielo.php?script=sci_arttext\&pid=S0001-37652016000200719

FISCH, G. Camada limite Amazônica: aspectos observacionais e de modelagem. 1996. 171f. Tese (Doutorado em Meteorologia) Instituto Nacional de Pesquisas Espaciais, São José Campos. http://www.scielo.br/scielo.php?script=sci_arttext\&pid=S0102-261X1999000100010

INMET. Normais Climatológicas do Brasil 1961-1990. (Org.) RAMOS, A. M. SANTOS, L. A. R. ; FORTES, L. T. G. 1. ed. Brasília: 2009. v. 1. 465p. http://www.inmet.gov.br/portal/index.php?r=clima/normaisClimatologicas 
MELLO, G. J.; PAULO, I. J. C.; PAULO, S. R.; GOMES, R. S. R.; MACHADO, N. G.; et al. Dimensão fractal de séries temporais medidas acima do dossel de floreta no Pantanal Mato-grossense. Revista Brasileira de Climatologia, v.12, p.61-83, $2013 \mathrm{~b}$. https://revistas.ufpr.br/revistaabclima/article/view/30937/21507

MELLO, G. J.; SILVEIRA, S. W. G.; GOMES, R. S. R.; MUSIS, C. R.; BIUDES, M. S.; et al. Análise sazonal da complexidade do saldo de radiação no Pantanal. Revista $\begin{array}{lllll}\text { Ciência e } & \text { Natura, } & \text { v.35, } & \text { p.86-97, } & \end{array}$ https://periodicos.ufsm.br/cienciaenatura/article/view/11215

MILLÁN, H.; KALAUZI, A.; LLERENA, G.; SUCOSHAÑAY J.; PIEDRA, D. Meteorological complexity in the Amazonian area of Ecuador: An approach based on dynamical system theory. Ecological Complexity, v.6, p.278-285, 2009. https://www.sciencedirect.com/science/article/pii/S1476945X09000555

NUNES DA CUNHA, C.; JUNK, W. J. Year-to-year changes in water level drive the of Vochysia divergens in Pantanal glassland. Applied Vegetation Science, v.7, p.103110, 2004. http://www.eventus.com.br/atbc2012/waterLevel_Vochysia.pdf

PAULO, S. R.; PAULO, I. J. C.; DECKER, Y. Reconstructing the micrometeorological dynamics of the southern Amazonian transitional Forest. Chaos: An Interdisciplinary Journal of Nonlinear Science, v. 25, n. 12, p. 123, 2015. https://aip.scitation.org/doi/10.1063/1.4938188

PARANHOS FILHO, A. C.; MOREIRA, E. S.; OLIVEIRA, A. K. M.; PAGOTTO, T. C. S.; MIOTO, C. L. Análise da variação da cobertura do solo no Pantanal de 2003 a 2010 através de sensoriamento remoto. Engenharia Sanitária e Ambiental, Rio de Janeiro, v. 19, n. especial, p. 69-76, 2014. http://www.scielo.br/scielo.php?pid=S1413$41522014000500069 \&$ script=sci_abstract\&tlng=pt

PINTO, E. G. F.; SAVI, M. A. Nonlinear prediction of time series obtained from an experimental pendulum. Current Topics in Acoustical Research - Research Trends, v.3, p.151-162, 2003. http://mecanon.coppe.ufrj.br/wpcontent/uploads/2017/08/PredPend_ResTrends03.pdf

SAVI, M. A. Dinâmica Não Linear e Caos. Rio de janeiro: Editora E-papers, 2006. 304p. http://www.e-papers.com.br/produtos.asp?codigo_produto=2929

SCHREIBER, T.; SCHMITZ, A. Surrogate times series. Physica D, v.142, p.346-382, 2000. https://www.sciencedirect.com/science/article/pii/S0167278900000439

SCHREIBER, T. Interdisciplinary application of nonlinear time series methods. Physics Reports, v.308, p.1-64, 1999. https://www.semanticscholar.org/paper/Interdisciplinaryapplication-of-nonlinear-time-

Schreiber/19bf5bdfb03791bb78ecb56e4483ce059258c5cd

SCHREIBER, T.; SCHMITZ, A. Improved surrogate data for nonlinearity tests. $\begin{array}{llll}\text { Physical Review } \quad \text { Letters, } & \text { v.77, } & \text { p.635-638, } & 1996 .\end{array}$ https://journals.aps.org/prl/abstract/10.1103/PhysRevLett.77.635 
WRIGHT, I. R.; NOBRE, C. A.; TOMASELLA, J.; ROCHA, H.R.; ROBERTS, J.M.; et al. Towards a GCM surface parameterization for Amazonia. In: GASH, J. H. C.; NOBRE, C. A.; ROBERTS, J. M.; VICTORIA, R. L. (eds.) Amazonian Deforestation and Climate. New York: J. M. Wiley and Sons, 1996, p.473-504. https://bdpi.usp.br/item/000921953 